\title{
Implementation of a neonatal platelet transfusion guideline to reduce non-indicated transfusions using a quality improvement framework
}

\author{
Patricia E. Davenport $\mathbb{D}^{1,2} \cdot$ Jenny Chan Yuen ${ }^{3} \cdot$ Julie Briere $^{1} \cdot$ Henry A. Feldman ${ }^{1,4} \cdot$ Martha C. Sola-Visner $^{1,2} \cdot$ \\ Kristen T. Leeman $\mathbb{1}^{1,2}$
}

Received: 9 December 2020 / Revised: 11 February 2021 / Accepted: 25 February 2021 / Published online: 23 March 2021

(c) The Author(s), under exclusive licence to Springer Nature America, Inc. 2021

\begin{abstract}
Objective Variation exists in neonatal platelet transfusion practices. Recent studies found potential harm in liberal platelet transfusion practices, supporting the use of lower transfusion thresholds. Our aim was to reduce non-indicated platelet transfusions through implementation of a restrictive platelet transfusion guideline.

Study design Platelet transfusions from January 2017 to December 2019 were classified as indicated or non-indicated using the new guideline. Interventions included guideline implementation and staff education. Outcomes were evaluated using statistical process control charts. Major bleeding was the balancing measure.

Result During study, 438 platelet transfusions were administered to 105 neonates. The mean number of non-indicated platelet transfusions/month decreased from 7.3 to 1.6. The rate of non-indicated platelet transfusions per 100 patient admissions decreased from 12.5 to 2.9. Rates of major bleeding remained stable.

Conclusions Implementation of a restrictive neonatal platelet transfusion guideline significantly reduced potentially harmful platelet transfusions in our NICU without a change in major bleeding.
\end{abstract}

\section{Introduction}

Thrombocytopenia, defined as a platelet count $<150 \times 10^{9} /$ L, affects $18-35 \%$ of all patients admitted to the Neonatal Intensive Care Unit (NICU). Its incidence is inversely proportional to the gestational age, reaching $\sim 70 \%$ among

These authors contributed equally: Martha C. Sola-Visner, Kristen T. Leeman

Supplementary information The online version contains supplementary material available at https://doi.org/10.1038/s41372021-01033-6.

Patricia E. Davenport

Patricia.Davenport@childrens.harvard.edu

1 Division of Newborn Medicine, Boston Children's Hospital, Boston, MA, USA

2 Harvard Medical School, Boston, MA, USA

3 Department of Pediatrics, Boston Children's Hospital, Boston, MA, USA

4 Institutional Centers for Clinical and Translational Research, Boston Children's Hospital, Boston, MA, USA extremely low birth weight infants (ELBW) with BW $<1000$ g [1-5].

Neonatal thrombocytopenia has been invoked as a potential contributor to the high incidence of intracranial hemorrhage ( $\mathrm{ICH}$ ) and bleeding among neonates. The desire to prevent bleeding has likely contributed to the widespread use of liberal platelet transfusion thresholds in neonates, with observational studies suggesting that most platelet transfusions in U.S. NICUs are given to non-bleeding neonates with platelet counts between 50 and $100 \times 10^{9} / \mathrm{L}$ [6]. However, there is a very poor relationship between the severity of thrombocytopenia and the incidence of bleeding, suggesting that factors other than the platelet count are better determinants of bleeding risk in neonates [7, 8]. Furthermore, several observational studies over the last decade reported a correlation between number of platelet transfusions and increased neonatal morbidity and mortality, even after adjustment for severity of illness [9-13].

Until recently, the paucity of high-quality evidence to guide clinical practice resulted in a lack of agreement regarding the platelet count below which a neonate should be transfused, which led to wide variability in transfusion practices $[14,15]$. Several surveys and observational studies 
revealed a striking inter-institutional and world-wide variability in neonatal platelet transfusion thresholds, with U.S. neonatologists generally transfusing at a higher platelet count threshold than European neonatologists [6, 14, 15]. In January of 2019, the PlaNet-2 trial was published. This large, multicenter, prospective trial randomized thrombocytopenic infants $<34$ weeks' gestation to receive platelet transfusions when the platelet count fell below $50 \times 10^{9} / \mathrm{L}$ (high threshold) or below $25 \times 10^{9} / \mathrm{L}$ (low threshold). The study found a significantly greater rate of death or major bleeding in the 28 days following randomization in neonates randomized to the high compared to the low threshold group (26\% vs. $19 \%$, respectively) [16]. Initial concerns about the generalizability of the findings to the highest risk neonates were largely alleviated by a recent sub-analysis of PlaNeT-2 data that evaluated for possible heterogeneity of treatment effect. After categorizing the infants according to their baseline risk of death or major bleeding, the investigators found that babies in all risk categories benefited from the lower platelet transfusion threshold [17].

While high-quality evidence like that provided by PlaNeT-2 is now available to guide platelet transfusion decisions in preterm neonates, data for term infants remain limited and largely derived from observational studies. Three prior retrospective studies investigated the association between platelet transfusions and mortality among NICU patients of all gestational and postnatal ages, and found an association between number of platelet transfusions and mortality [9, 10, 13]. Taken together, this body of evidence strongly suggest that thoughtful, evidence-based decision making regarding platelet transfusions in preterm as well as full-term neonates and infants is essential, and that current liberal transfusion practices in neonatology should be revisited.

\section{Methods}

\section{Setting}

The Boston Children's Hospital NICU is an academic tertiary and quaternary referral center serving infants $<6$ months corrected age with complex medical or surgical conditions. More than 650 outborn infants are admitted each year, transferred from other hospitals or through the emergency room. More than $80 \%$ of these infants are $<1$ month postnatal age at admission. All infants are cared for by a multidisciplinary team, including neonatologists, neonatal fellows, nurse practitioners, bedside nurses, respiratory therapists, nutritionists, and pharmacists. The team caring for infants with surgical diagnoses additionally includes pediatric surgeons, pediatric surgical fellows, and surgical critical care fellows.
Before this project, providers made decisions about when to transfuse platelets based on a previously published guideline that advised using a platelet count $<50 \times 10^{9} / \mathrm{L}$ as a transfusion threshold based on factors such as illness severity, coagulopathy, pre- or postoperative status, and low birth weight/early postnatal age [18]. Under this guideline, higher platelet counts were used as transfusion thresholds in sicker or clinically unstable neonates, in hopes of decreasing their bleeding risk. The growing evidence that liberal platelet transfusions may not decrease bleeding risk and may instead be harmful to neonates had begun to change individual provider practice, but significant variation still existed, mirroring the practice variation described across NICUs in the USA [6].

\section{Interventions}

A multidisciplinary team developed a key driver diagram (Fig. 1) to plan interventions to achieve our specific aim of minimizing non-indicated platelet transfusions from a baseline of 7 to $<3$ per month over 1 year. Plan-Do-StudyAct (PDSA) cycles were conducted to test effectiveness of interventions.

\section{Guideline development}

The team created a practice guideline based on a review of existing evidence and expert opinion that recommended platelet transfusion thresholds based on gestational age, chronological age, and specific risk factors. Surgery and neurosurgery teams advised perioperative thresholds based on their expertise. Infants at the highest risk of ICH (those $<28$ weeks' gestation and $\leq 7$ days old) were placed in a higher transfusion threshold category due to some uncertainty regarding the translatability of PlaNeT-2 findings to these patients. Specifically, since the average time of randomization in the trial was day of life 7 , and $39 \%$ of infants received a platelet transfusion prior to randomization, it was possible that these transfusions were given during the highest risk period for ICH. Additionally, all infants in the trial received a point of care (POC) bedside head ultrasound within $6 \mathrm{~h}$ prior to randomization to ensure the absence of ICH. Currently, our unit staff is not trained to perform POC bedside ultrasounds, thus preventing the rapid assessment for new/evolving ICH prior to transfusion decisions. Together, this created enough uncertainty in this high-risk group that they were assigned to a more liberal transfusion threshold of $<50 \times 10^{9} / \mathrm{L}$. For all non-bleeding neonates outside of this high-risk group/period (unless they had a major bleed in the past $48 \mathrm{~h}$, were immediately prior to surgery, or immediately pre/post-op from a major neurosurgical procedure) a platelet transfusion threshold of $<25 \times$ $10^{9} / \mathrm{L}$ was recommended regardless of severity of illness or 
Fig. 1 Key Driver Diagram. Diagram outlining the projectspecific aim, key drivers, and change concepts.
Table 1 Neonatal platelet transfusion guidelines before (version 1) and after (version 2) January 2019.

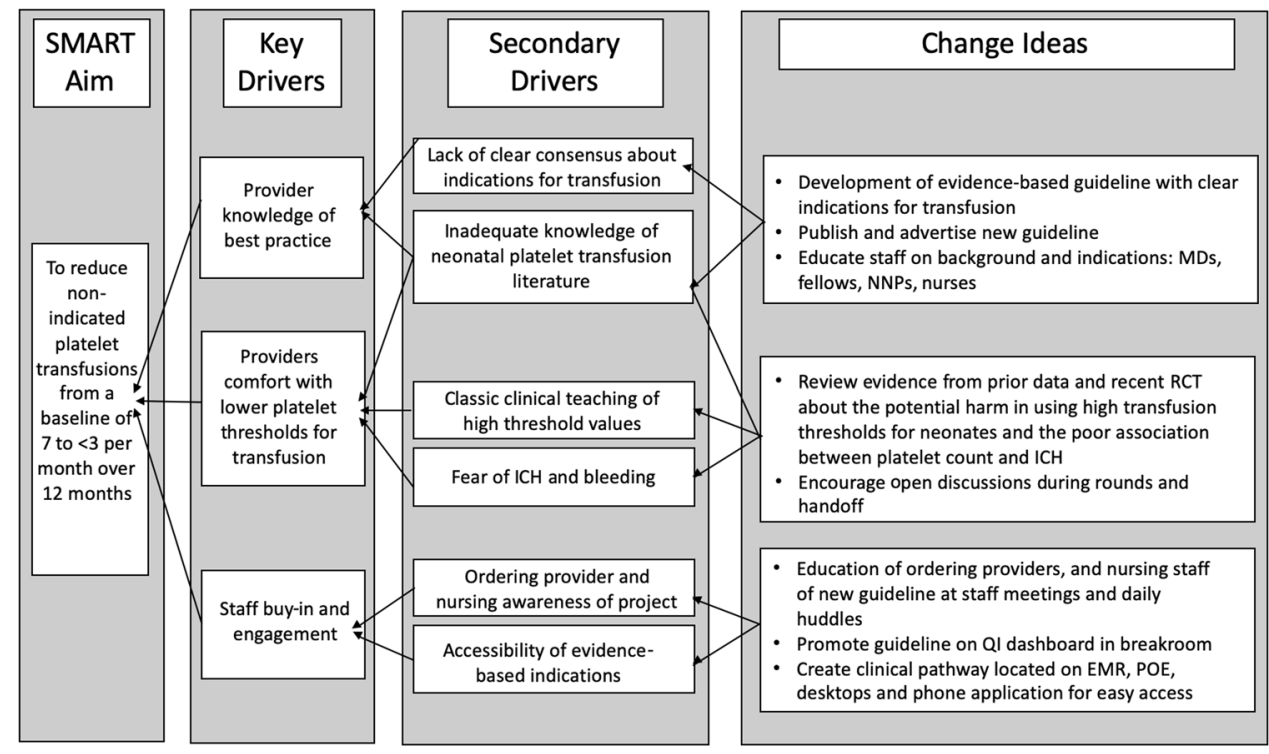

\begin{tabular}{|c|c|c|}
\hline & $\begin{array}{l}\text { Platelet transfusion guideline } \\
\text { version } 1 \text { thresholds }^{\mathrm{a}} \\
\left(\times 10^{9} / \mathrm{L}\right)\end{array}$ & $\begin{array}{l}\text { Platelet transfusion guideline } \\
\text { version } 2 \text { thresholds }^{\mathrm{a}} \\
\left(\times 10^{9} / \mathrm{L}\right)\end{array}$ \\
\hline Active bleeding & Transfuse & Transfuse \\
\hline Transfuse all & $<30$ & $<25$ \\
\hline $\begin{array}{l}\text { Clinical instability (i.e., vasopressor } \\
\text { requirement, high ventilator settings) }\end{array}$ & $<50$ & $<25$ \\
\hline \multicolumn{3}{|l|}{ Prematurity } \\
\hline $\mathrm{BW}<1500 \mathrm{~g}$ and $<7$ days & $<50$ & N/A \\
\hline$<28$ weeks GA and $<7$ days & N/A & $<50$ \\
\hline Concurrent coagulopathy & $<50$ & $<25$ \\
\hline Prior significant hemorrhage & $\begin{array}{l}<50 \text { (regardless of time } \\
\text { since bleed) }\end{array}$ & $\begin{array}{l}<50 \text { (if bleeding within } \\
\text { last } 48 \mathrm{~h} \text { ) }\end{array}$ \\
\hline Platelet transfusion volume & Unspecified (usually $15 \mathrm{ml} / \mathrm{kg}$ ) & $10 \mathrm{ml} / \mathrm{kg}$ \\
\hline Platelet transfusion rate & $\begin{array}{l}\text { Unspecified (usually over } \\
30-60 \text { min) }\end{array}$ & Over $2-3 \mathrm{~h}$ \\
\hline
\end{tabular}

${ }^{\mathrm{a}}$ These recommendations are based on medical evidence and professional expert opinions. Decisions about treatment are the responsibility of the treating clinician and should be tailored to individual circumstances. Platelet volume and transfusion time are provided for reference only. Providers should refer to institutional formulary or guidelines when prescribing. coagulopathy. The latter represented the most significant departure from our group's prior practice of transfusing clinically unstable neonates at higher platelet counts. This guideline was made available in January 2019. Key differences between the two guidelines are outlined in Table 1 .

\section{Multipronged staff educational efforts}

After guideline development, roll out efforts and education began as a project launch from January 2019 to July 2019. Staff education occurred in several ways. In February 2019, the team discussed the background, evidence, and new guideline at a faculty meeting. In March and April 2019, fellows and neonatal nurse practitioners were educated. Targeted group education occurred throughout the launch period at daily huddles to increase staff awareness of new guideline recommendations. One-on-one training occurred via discussions on rounds and with providers as questions arose regarding transfusion indications.

\section{Encouraging staff buy-in}

Throughout the study period, the team used the multidisciplinary daily operations brief forum to update all 
disciplines about the guideline and remind them of the project. During sign out, QI team members encouraged providers to discuss platelet transfusion thresholds for "at risk" babies to ensure team consensus on parameters and address any questions. Project progress was also promoted on the electronic QI board in the staff breakroom to advertise the importance of the guideline.

\section{Creation of an electronic clinical pathway}

An electronic clinical pathway was developed to increase applicability and generalizability of the guideline to neonates admitted to other areas of the hospital (cardiac and pediatric intensive care units, and emergency room), and to increase overall accessibility and usability of the guideline. Through an iterative process with platelet experts, QI experts, and hospital wide stakeholders, the clinical pathway was developed and made available in October 2019. It is accessible in several locations, including the electronic medical record and the Clinical Pathways mobile and desktop application present on every hospital computer. Clinicians can access this pathway easily when making decisions about platelet transfusions.

\section{Data collection and measures}

A retrospective chart review was performed from January 2017 to December 2018 to obtain baseline data on rate and indications of platelet transfusions. To do this, a complete list of all platelet transfusions administered in the NICU was obtained from the Blood Bank and patient charts were reviewed to determine the platelet count at time of transfusion and the indication. We then prospectively collected the same data for 12 months after project start. We obtained census, demographic data, and data on bleeding events from the medical record.

The primary outcome was the number of platelet transfusions that did not meet the new guideline criteria (i.e., non-indicated transfusions) per month. Secondary outcomes included the percentage of patients who received a non-indicated platelet transfusion, the rate of nonindicated transfusions per 100 NICU admissions, and the total number of platelet transfusions (both indicated and non-indicated). As a balancing measure, the rate of major bleeding was compared in the pre- and post-guideline periods. Major bleeding was defined as any grade of ICH, pulmonary hemorrhage, upper gastrointestinal bleeding, rectal bleeding, adrenal hemorrhage, or non-specified hemorrhages. Data on major bleeding were obtained from the medical records. ICHs that occurred prior to admission to our hospital and remained stable during the hospitalization were excluded.

\section{Statistical analysis}

We used statistical process control (SPC) charts to analyze our measures over time and determine whether significant changes occurred with our interventions [19]. SQCpack software was used to create SPC charts for QI analysis. For the demographic data, Wilcoxon rank-sum and chi-squared tests were used to determine whether differences between pre-intervention and post-intervention populations existed. The percentages of non-indicated transfusions pre- and post-intervention were compared by logistic regression, and the rates per month and per admission were compared by Poisson regression.

\section{Ethics}

This project was identified by the Boston Children's Hospital Institutional Review Board as a quality improvement project and was therefore exempt. This manuscript was written using the SQUIRE guidelines [20].

\section{Results}

During the study period, 438 platelet transfusions were administered to 105 neonates. All transfused platelet units were leukoreduced and irradiated, but not pathogen reduced. There were no significant differences in demographic characteristics of the population before and after guideline implementation (Table 2). Most infants were $\geq 37$ weeks' gestation with birth weight $>2500$ grams. No differences in the rates of sepsis, necrotizing enterocolitis, thrombosis, or overall mortality were found. The number of non-indicated platelet transfusions per month decreased from a mean of 7.3 before to 1.6 after guideline implementation, with significant special cause variation on c-chart SPC analysis (Fig. 2A), and also with significance in pre- versus post-intervention analysis, $p<0.0001$ (Table 3). The percentage of transfusions that were non-indicated significantly decreased from 49 to $24 \%$ after project start $(p=0.0001)$ (Table 3). The percentage of neonates per 100 admissions who received one or more non-indicated platelet transfusions decreased from $4.4 \%$ pre-guideline to $1.7 \%$ post-guideline, $p<0.001$ (Table 3). The rate of nonindicated platelet transfusions per 100 patient admissions also decreased from 12.5 to 2.9 (Fig. 2B), $p<0.0001$ (Table 3). When examining the total number of transfusions (indicated and non-indicated), we also found a significant reduction from 15.5 to 6.6 platelet transfusions per month, $p<0.0001$ (Table 3). Analysis of balancing measures showed that rates of major bleeding, including $\mathrm{ICH}$, remained stable over time (Table 4). A full description of patients who developed or had progression of ICH during 
Table 2 Characteristics of infants admitted to the NICU during each period of the project.
A

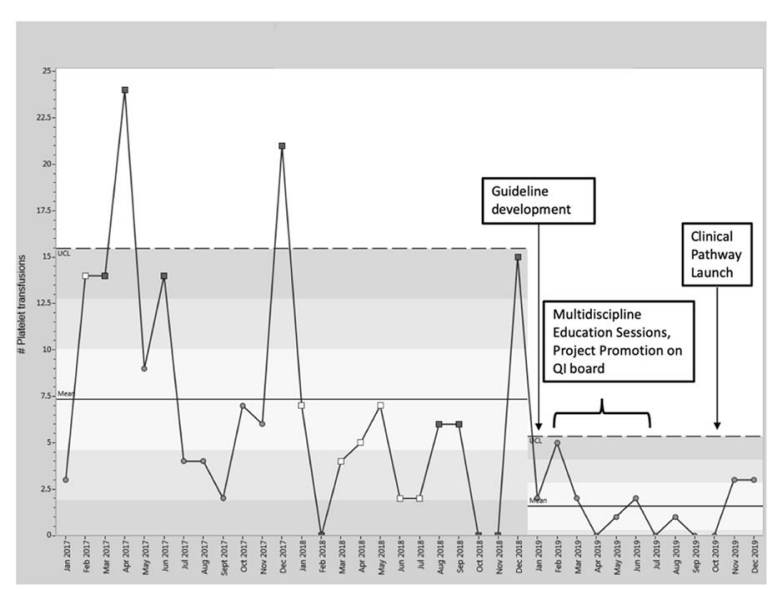

Fig. 2 Significant decreases in non-indicated platelet transfusions after project start on SPC analysis. Special cause variation shows significant change. A Decreased number of non-indicated platelet transfusions/month after project start from 7.3 to 1.6 on SPC C-chart
B

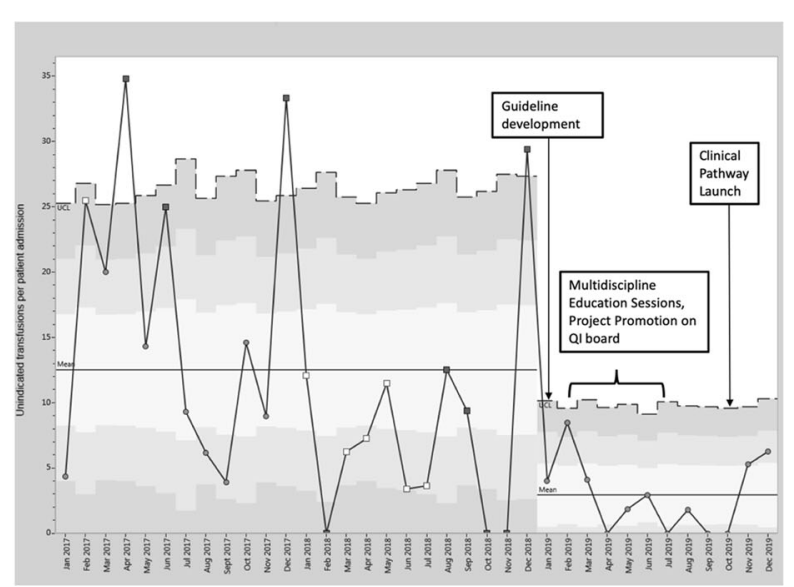

analysis. B Rate of non-indicated transfusions per 100 patient admissions decreased from 12.5 to 2.9 after project start on U-chart analysis. For both $\mathbf{A}$ and $\mathbf{B}$, the rule used to determine special cause variation was 8 or more points below the centerline. 
Table 3 Outcome and process measures.

\begin{tabular}{llllc}
\hline & $\begin{array}{l}\text { Pre-guideline } \\
(2017-2018)\end{array}$ & $\begin{array}{l}\text { Post- } \\
\text { guideline }(2019)\end{array}$ & $\begin{array}{l}\text { Ratio, } \\
\text { Post: Pre }\end{array}$ & $p$ \\
\hline Months & 24 & 12 & & \\
Admissions & 1407 & 666 & $0.33^{\mathrm{a}}$ & 0.0001 \\
Transfusions & 359 & 79 & & \\
$\quad 183(51 \%)$ & $60(76 \%)$ & & $<0.0001$ \\
$\quad$ Indicated & $176(49 \%)$ & $19(24 \%)$ & $0.44^{\mathrm{c}}$ & $<0.0001$ \\
$\quad$ Non-indicated & $15.0 \pm 0.8^{\mathrm{b}}$ & $6.6 \pm 0.7$ & 0.66 & $<0.0001$ \\
Transfusions per month & $7.6 \pm 0.6$ & $5.0 \pm 0.6$ & 0.22 & 0.01 \\
$\quad$ Indicated & $7.3 \pm 0.6$ & $1.6 \pm 0.4$ & 0.46 & $<0.0001$ \\
$\quad$ Non-indicated & $25.5 \pm 1.4$ & $11.9 \pm 1.3$ & 0.69 & \\
Transfusions per 100 admissions & $13.0 \pm 1.0$ & $9.0 \pm 1.2$ & 0.23 & \\
$\quad$ Indicated & $12.5 \pm 0.9$ & $2.9 \pm 0.7$ & & 0.002 \\
$\quad$ Non-indicated & 62 & 11 & 0.35 & 0.003 \\
Patients with non-indicated & & & 0.37 & \\
transfusion & $2.6 \pm 0.3$ & $0.9 \pm 0.3$ & $1.7 \pm 0.5$ & \\
$\quad$ Per month & $4.4 \pm 0.6$ & Per 100 admissions &
\end{tabular}

${ }^{a}$ Odds ratio $(\mathrm{OR})$ for non-indicated transfusion, from logistic regression. $p$ tests $\mathrm{H}_{0}$ : $\mathrm{OR}=1$.

${ }^{\mathrm{b}}$ Rate \pm standard error, from Poisson regression.

${ }^{c}$ Rate ratio (RR) from Poisson regression. $p$ tests $\mathrm{H}_{0}: \mathrm{RR}=1$.

\begin{tabular}{lccl}
\hline & $n(\%)$ & $p^{\text {a }}$ \\
\cline { 2 - 3 } & Pre-intervention January & $\begin{array}{l}\text { Post-intervention January } \\
2019-\text { December 2019 }\end{array}$ & 0.65 \\
\hline Admissions & $2017-$ December 2018 & 687 \\
Intracranial & 1388 & $9(1.3)$ & \\
hemorrhage, total & $22(1.6)$ & $7(1.0)$ \\
Grade I IVH & $13(0.9)$ & $1(0.1)$ \\
Grade II IVH & $3(0.2)$ & $0(0.0)$ \\
Grade III IVH & $2(0.1)$ & $1(0.1)$ \\
Intraparenchymal & $4(0.3)$ & $37(5.5)$ \\
Other bleeding, total & $107(7.7)$ & $1(0.1)$ \\
Pulmonary hemorrhage & $7(0.5)$ & $3(0.4)$ \\
Upper GI bleeding & $7(0.5)$ & $2(0.3)$ \\
Rectal bleeding & $4(0.3)$ & $1(0.1)$ \\
Adrenal hemorrhage & $1(0.1)$ & $30(4.4)$ \\
Non-specified & $88(6.3)$ & \\
hemorrhage & &
\end{tabular}

Totals compared by chi-squared test, corroborated by Fisher exact test.

Table 4 Balancing measure: incidence of major bleeding, per site.

their hospitalization in our unit is provided (Supplementary Table 1).

\section{Discussion}

The creation and implementation of a restrictive neonatal platelet transfusion guideline resulted in a rapid and significant decrease in the number of non-indicated transfusions in our tertiary and quaternary NICU. We also showed a significant reduction in total number of platelet transfusions administered despite no significant change in unit admissions, demographics, or disease burden, suggesting it was attributable to practice change. The number of non-indicated platelet transfusions declined quickly after guideline implementation, suggesting that education alone was enough to change practice. We attribute this to multiple factors. First, educating providers on the evidence that 
liberal prophylactic platelet transfusions do not decrease a neonate's bleeding risk helped reduce platelet transfusions administered in hopes of preventing bleeding. Second, the high-quality evidence from the PlaNet- 2 trial showing that liberal platelet transfusion practices increased neonatal mortality motivated providers to seek out practice change and resulted in rapid and widespread acceptance of the new restrictive guideline.

After guideline launch, interventions included extensive staff education and development of an accessible electronic clinical pathway. Monthly data collection was key to the project's success by allowing identification of clinical scenarios that needed additional thought and/or clarification. Key footnotes were added to the clinical pathway to specify recommendations based on common questions, including defining minor bleeding, clarifying recommendations for non-bleeding patients with coagulopathy, and providing recommendations for actively anticoagulated neonates. We also noted an increase in documentation of both the platelet transfusion threshold and the reason for a platelet transfusion during the study period, suggesting both increased awareness of and adherence to the guideline. Consistent with our expectations at the time of guideline development, the biggest reduction in nonindicated platelet transfusions came from withholding transfusions in non-bleeding, critically ill neonates with platelet counts between 25 and $50 \times 10^{9} / \mathrm{L}$, who were historically transfused at the higher threshold of $50 \times 10^{9} / \mathrm{L}$ due to their severity of illness. In agreement with previous trial data, despite the decrease in platelet transfusion thresholds and decrease in non-indicated platelet transfusions, we did not see a change in the incidence of major bleeding, including ICH.

Our project has some limitations. First, our implementation interventions may not be generalizable to NICUs that care for a different patient mix (i.e., nonsurgical cases or primarily inborn premature infants) or those where more restrictive platelet transfusion practices are already in place. Our relatively older neonatal population with a lower number of infants at highest risk of ICH (ELBW infants $<72 \mathrm{~h}$ old) may have made practice change easier. However, our guideline was not designed with our specific unit's population in mind, but rather with the intention to serve as a template for more restrictive transfusion approaches applicable to typical NICU patients. In NICUs with different patient populations, additional interventions and PDSA cycles may need to be tailored to specific unit culture and context to change practice.

In a young field such as neonatology there are inherently many unanswered clinical questions and new research will continue to challenge accepted practice to improve the care of this vulnerable population. There is a growing understanding that, in addition to their well-known hemostatic functions, platelets play important roles in the regulation of immune responses, inflammation, and angiogenesis [21, 22], and it is possible that some of the effects of platelet transfusions are mediated by their non-hemostatic functions. With a better understanding of the diverse functions of platelets and the findings of PlaNeT-2, it has become increasingly evident that platelet transfusions may have harmful effects in neonates, and that platelet transfusion decisions in this population should be done judiciously. While researchers work to elucidate the biology underlying these findings, it is our job as neonatologists to critically evaluate and review new evidence and to change practices based on the best available data. Education and adherence to evidence-based guidelines will likely result in a reduction in unnecessary platelet transfusions and improved outcomes.

\section{Conclusion}

Through a quality improvement framework, implementation of a neonatal platelet transfusion guideline reduced nonindicated platelet transfusions without a change in major bleeding. High impact evidence was swiftly translated into direct clinical change to improve patient care. PDSA cycles focused on multidisciplinary education, fostering of buy-in and improving accessibility via clinical pathway creation led to successful practice change. This work is generalizable to similar inpatient settings to reduce unnecessary and potentially harmful platelet transfusions.

Author contributions PED conceptualized and designed the study, collected data, carried out the analyses, drafted the initial manuscript, and reviewed and revised the manuscript. JCY contributed to the guideline development, data analysis, and reviewed and revised the manuscript. JB contributed to project implementation and reviewed and revised the manuscript. HAF contributed to data analysis and reviewed and revised the manuscript. MCS-V and KTL conceptualized and designed the study, contributed to data analysis, and reviewed and revised the manuscript. All authors approved the final manuscript as submitted and agree to be accountable for all aspects of the work.

Funding PED's work was supported by the National Heart, Lung, and Blood Institute (NHLBI) of the National Institute of Health under grant number T32HL007917-21 (PI: Robert C. Flaumenhaft).

\section{Compliance with ethical standards}

Conflict of interest The authors declare no competing interests.

Publisher's note Springer Nature remains neutral with regard to jurisdictional claims in published maps and institutional affiliations.

\section{References}

1. Andrew M, Castle V, Saigal S, Carter C, Kelton JG. Clinical impact of neonatal thrombocytopenia. J Pediatrics. 1987;110:457-64. 
2. Castle V, Andrew M, Kelton J, Giron D, Johnston M, Carter C. Frequency and mechanism of neonatal thrombocytopenia. J Pediatrics. 1986;108:749-55.

3. Mehta P, Vasa R, Neumann L, Karpatkin M. Thrombocytopenia in the high-risk infant. J Pediatrics. 1980;97:791-4.

4. Christensen RD, Henry E, Wiedmeier SE, Stoddard RA, Sola-Visner MC, Lambert DK, et al. Thrombocytopenia among extremely low birth weight neonates: data from a multihospital healthcare system. J Perinatol. 2006;26:348-53.

5. Sola-Visner M, Sallmon H, Brown R. New insights into the mechanisms of nonimmune thrombocytopenia in neonates. Semin Perinatol. 2009;33:43-51.

6. Sparger KA, Assmann SF, Granger S, Winston A, Christensen $\mathrm{RD}$, Widness JA, et al. Platelet transfusion practices among verylow-birth-weight infants. JAMA Pediatrics. 2016;170:687-94.

7. Muthukumar P, Venkatesh V, Curley A, Kahan BC, Choo L, Ballard $\mathrm{S}$, et al. Severe thrombocytopenia and patterns of bleeding in neonates: results from a prospective observational study and implications for use of platelet transfusions. Transfus Med. 2012;22:338-43.

8. Deschmann E, Saxonhouse MA, Feldman HA, Norman M, Barbian M, Sola-Visner M. Association of bleeding scores and platelet transfusions with platelet counts and closure times in response to adenosine diphosphate (CT-ADPs) among preterm neonates with thrombocytopenia. JAMA Netw Open. 2020;3:e203394.

9. Del Vecchio A, Sola MC, Theriaque DW, Hutson AD, Kao KJ, Wright $\mathrm{D}$, et al. Platelet transfusions in the neonatal intensive care unit: factors predicting which patients will require multiple transfusions. Transfusion. 2001;41:803-8.

10. Garcia MG, Duenas E, Sola MC, Hutson AD, Theriaque D, Christensen RD. Epidemiologic and outcome studies of patients who received platelet transfusions in the neonatal intensive care unit. J Perinatol. 2001;21:415-20.

11. Bonifacio L, Petrova A, Nanjundaswamy S, Mehta R. Thrombocytopenia related neonatal outcome in preterms. Indian J Pediatr. 2007;74:269-74.
12. Kenton AB, Hegemier S, Smith EO, O'Donovan DJ, Brandt ML, Cass DL, et al. Platelet transfusions in infants with necrotizing enterocolitis do not lower mortality but may increase morbidity. J Perinatol. 2005;25:173-7.

13. Baer VL, Lambert DK, Henry E, Snow GL, Sola-Visner MC, Christensen RD. Do platelet transfusions in the NICU adversely affect survival? Analysis of 1600 thrombocytopenic neonates in a multihospital healthcare system. J Perinatol. 2007;27:790-6.

14. Josephson CD, Su LL, Christensen RD, Hillyer CD, Castillejo MI, Emory MR, et al. Platelet transfusion practices among neonatologists in the United States and Canada: results of a survey. Pediatrics. 2009;123:278-85.

15. Cremer M, Sola-Visner M, Roll S, Josephson CD, Yilmaz Z, Buhrer $\mathrm{C}$, et al. Platelet transfusions in neonates: practices in the United States vary significantly from those in Austria, Germany, and Switzerland. Transfusion. 2011;51:2634-41.

16. Curley A, Stanworth SJ, Willoughby K, Fustolo-Gunnink SF, Venkatesh V, Hudson C, et al. Randomized trial of platelettransfusion thresholds in neonates. N Engl J Med. 2019;380:242-51.

17. Fustolo-Gunnink SF, Fijnvandraat K, van Klaveren D, Stanworth S, Curley AE, Onland W, et al. Preterm neonates benefit from low prophylactic platelet transfusion threshold despite varying risk of bleeding or death. Blood. 2019;134:2354-60.

18. Sparger K, Deschmann E, Sola-Visner M. Platelet transfusions in the neonatal intensive care unit. Clin Perinatol. 2015;42:613-23.

19. Provost L, Murray, S. The health care data guide: learning from data for improvement. San Francisco, CA: Jossey-Bass; 2011.

20. Ogrinc G, Davies L, Goodman D, Batalden P, Davidoff F, Stevens D. SQUIRE 2.0 (Standards for QUality Improvement Reporting Excellence): revised publication guidelines from a detailed consensus process. J Nurs Care Qual. 2016;31:1-8.

21. Weyrich AS. Platelets: more than a sack of glue. Hematol Am Soc Hematol Educ Program. 2014;2014:400-3.

22. Sola-Visner M, Bercovitz RS. Neonatal platelet transfusions and future areas of research. Transfus Med Rev. 2016;30:183-8. 